\title{
The Analysis of Characteristics of Aviation AC Series Fault Arc
}

\author{
Feng Zhong a , Junmin Zhang ${ }^{b}$ \\ School of Automation Science and Electrical Engineering, Beihang University, \\ Beijing 100191, China \\ asdzf91@163.com, bjmzhang@buaa.edu.cn
}

\begin{abstract}
The AC series fault arc tests are conducted by a self-design platform according to several common test standards for fault arc circuit breakers in this paper. Compared with the no-arc current waveform, the current of fault circuit has shoulders when the current passes zero, and the current of fault circuit is lower than the no-arc current. A united method of the wavelet transform (WT) and the information entropy (IE) is proposed to distinguish the fault and normal circuit. By the WT of arc current and the analysis of multiple-sampling, the results reveal that the arc fault frequency bands (FB) range is $(625,1250) \mathrm{Hz}$. The wavelet component D3 of arc current and its wavelet energy (WE) have large value during the arc period. The wavelet energy of no-arc circuit is lower than 0.006 . The IE is bigger than 1 in the fault circuit. Therefore, the FB, WE, IE can be chosen as the fault characteristics to detect the fault circuits.
\end{abstract}

Keywords: Arc fault; Fault detection; Wavelet transform; Wavelet energy; Information entropy.

\section{Introduction}

As the aircraft ages, the number of arc faults in cables will increase. Moreover the special flight environment also have effects on the fault arc appearances. For arc duration is short and the current is small, the fault cannot detect by the aviation circuit breaker quickly. When the arc fault is serious, it causes the fire and power system fault, even more a casualty tragedy, which is a great threat to aviation safe. Therefore it is necessary to detect the aviation arc fault for the safety of aviation [1-3].

The common types of arc faults are series arc fault and parallel arc fault. The series arc fault results from the loose connection in the wires, which is shown in Fig. (1-a) [4]. Its fault current is small. Parallel fault occurs between phase and neutral line or phase line and phase line, shown in figure (1-b). It caused by two adjacent wires short-circuit, which results from two or more wires insulation damaging, and the metal conductor exposed [5]. It is more dangerous and releases even greater energy than the series arc fault.

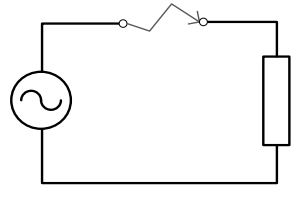

(a)

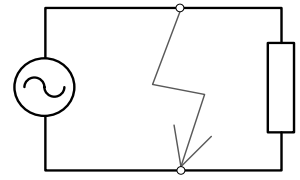

(b)

Fig. 1 The types of arc fault

Recently, most of the authors pay attention to research of the fault arc. In Refs [6], the author uses weight direct determined neural network method to recognize arc fault. In Refs [7], the author applies The wavelet neural network method and by changing the arc faults of different loads to verify its accuracy. In Refs [8], the author uses wavelet packets and proposes a new algorithm to distinguish the arc faults. In Refs [5], the author pays attention to arc characteristics of different loads and with the help arc-fault circuit interrupter (AFCI), the fault of different types can be detected.

According to a number of common test standards of fault arc circuit breakers, the AC series arc fault platform are established and the current signal is sampled. A united method of the wavelet transform (WT) and the information entropy (IE) is proposed to distinguish the fault and normal circuit. Comparing the similarities and differences between the fault current signal and the normal current signal, a relatively reliable distinguishing feature of fault is proposed. 


\section{The Experimental device}

Nowadays, the aircraft electrical systems operate at $115 \mathrm{~V} \mathrm{AC}(400 \mathrm{~Hz})$. So the paper uses the $115 \mathrm{~V}, 400 \mathrm{~Hz}$ power supply to research the arc fault, ignoring the effect of air pressure during the aviation flight. Referencing to a number of internationally common test standards for fault arc circuit breakers $[9,10]$, the series arc generator is designed in Fig. 2.

The circuit elements mainly include a AC $115 \mathrm{~V}(400 \mathrm{~Hz})$ power supply, a switch $\mathrm{K}$, different types of resistance $\mathrm{Z}$, a oscilloscope, a clamp current transducer $\mathrm{T}$ and the series arc generator device $\mathrm{M}$.

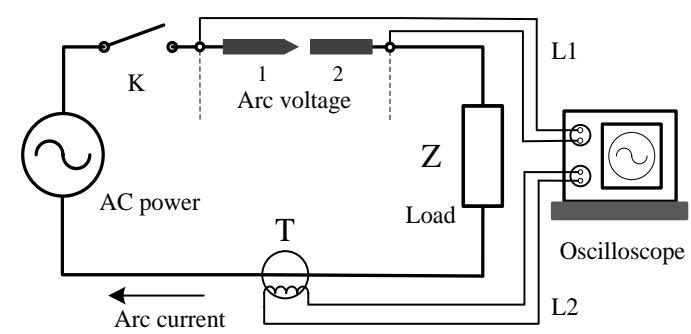

Fig. 2 The experimental circuit of series arc

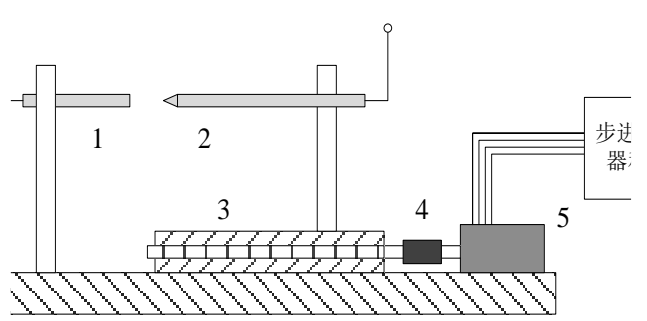

Fig. 3 Series arc generator

The series arc generator device is shown in Fig. 3. In the picture, the fixed contact 1 is fixed electrode. The moving electrode, contact 2 , with a sharp head is controlled by a stepper motor. The motor drives screw guide to control the velocity of moving electrode, which can simulate the series arc fault caused by a loose connection of the wire. In order to avoid the occasion of experiment, the experiment should be repeated more than 30 times for each kind of load.

\section{The analysis method of arc fault}

According the previous researches, the arc current contains high frequency components and it change over time irregularly. So the paper uses wavelet transform (WT).

\subsection{Wavelet Transform.}

The wavelet transform (WT) is defined as $[11,12]$

$W T_{f}(a, \tau)=\frac{1}{\sqrt{a}} \int_{R} f(t) \psi^{*}\left(\frac{t-\tau}{a}\right) d t$

Where, $f(t) \in L^{2}(R)$ and $\psi(t)$ is the wavelet function.

$\psi_{a, \tau}(t)$ is defined as

$\psi_{a, \tau}(t)=\frac{1}{\sqrt{a}} \psi\left(\frac{t-\tau}{a}\right), a>0, \tau \in R$

Where, $a$ is the dilation factor and $\tau$ is the translation factor.

Through wavelet transform the signal is divided into different frequency bands. The signal $x(t)$ is discretized to sample time series $x(n)[13], J$ is the layers of Wavelet decomposition.

$$
\begin{aligned}
x(n) & =D_{1}(n)+A_{1}(n)=D_{1}(n)+D_{2}(n)+A_{2}(n) \\
& =\sum_{j=1}^{J} D_{j}(n)+A_{J}(n)
\end{aligned}
$$

At each layer $j$, approximation signal $A_{j}$ and detail signal $D_{j}$ can be created. The signal $A_{j}$ will be decomposed into approximation signal $A_{j+1}$ and detail signal $D_{j+1}$. The whole process is equivalent to a signal $A_{j}$ passing through a high-pass filter and low-pass filter. The signal $A_{j+1}$ is the low-frequency signal and the signal $D_{j+1}$ is the high-frequency signal.

$A_{J}(n)$ is the $J^{\text {th }}$ layer approximation signal.

The frequency bands (FB) range of every component is as follows:

$$
\left\{\begin{array}{l}
D_{j}(n):\left[2^{-(j+1)} f_{s}, 2^{-j} f_{s}\right] \\
A_{j}(n):\left[0,2^{-(j+1)} f_{s}\right]
\end{array} \quad j=1,2, \cdots, \quad J\right.
$$

In the equation, $f_{s}$ is the sampling frequency, $j$ is the number of layer decomposition, $n$ is the nth sampling point. In this paper, the sampling frequency of the oscilloscope is $5000 \mathrm{~Hz}$. 
The paper uses 3 layer of wavelet decomposition with the mother wavelet of Daubechies 4(db4) that is widely used in waveform analysis.

\subsection{Wavelet Energy.}

On the basis of wavelet transform theory, the wavelet energy of sampling points is

$$
E(n)=\sqrt{\sum_{j=1}^{J} E_{j}(n)}=\sqrt{\sum_{j=1}^{J}\left|D_{j}(n)\right|^{2}}
$$

Where, $n$ is the $n^{\text {th }}$ sampling point and $D_{j}(n)$ is the reconstructing signal of $j^{\text {th }}$ layer.

\subsection{The Information Entropy Principle.}

Shannon's entropy is an important uncertainty measurement for evaluating and analyzing data. It is defined as follows:

Set the vector $\boldsymbol{X}$ as a discrete random variable with n possible states, Set the vector $\boldsymbol{p}$ as those $n$ states appearance probability.

Where, $\boldsymbol{X}=\left\{x_{1}, x_{2}, x_{3}, \ldots x_{n}\right\}, \boldsymbol{p}=\left\{p_{1}, p_{2}, p_{3}, \ldots p_{n}\right\}$, the values of $\boldsymbol{p}$ satisfy the terms of

$$
\left\{\begin{array}{l}
0 \leq p_{i} \leq 1 \\
\sum_{i=1}^{n} p_{i}=1
\end{array}\right.
$$

The definition of information entropy (IE) proposed by Shannon is as follows [14, 15]:

$$
I E=H\left(p_{1}, p_{2}, \ldots, p_{3}\right)=-k \sum_{i=1}^{n} p_{i} \log _{a} p_{i}
$$

Where, the $H(x)$ is the information entropy which is a measurement expressing the uncertainty by distribution of probability. In the formula, the base-a can be any positive number. Under normal circumstance, $a$ is chosen to 2 and the $k$ is chosen to 1 , therefore, the paper does too.

The arc current has the uncertain magnitude and high frequency harmonics components, whereas the normal current has a constant range, so the arc current has a bigger information entropy than normal current. $E N$ is 0.006 that is threshold distinguishing the arc from no-arc circuit. The wavelet energy range is divided into $n$ regions and $n=8$. The energy section is

$$
\left(0, \frac{E N}{n}\right], \ldots \ldots,\left(\frac{(n-1) E N}{n}, E N\right],(E N,+\infty)
$$

Calculate the probability of wavelet energy of each sampling point in the 50 points in the above 9 region, then solve the information entropy.

\section{Experimental Results and Analysis}

The loads current is $1 \mathrm{~A}, 5 \mathrm{~A}, 10 \mathrm{~A}$, respectively. The arc burn with a bright flame in Fig. 4. Because of the same change rule of arc current when the fault occurs, the paper takes arc faults under 115V $1 \mathrm{~A}$ condition as an example to analyze the fault arc characteristics, which is shown in Fig. 5. Fig. 6 is the WT of current.

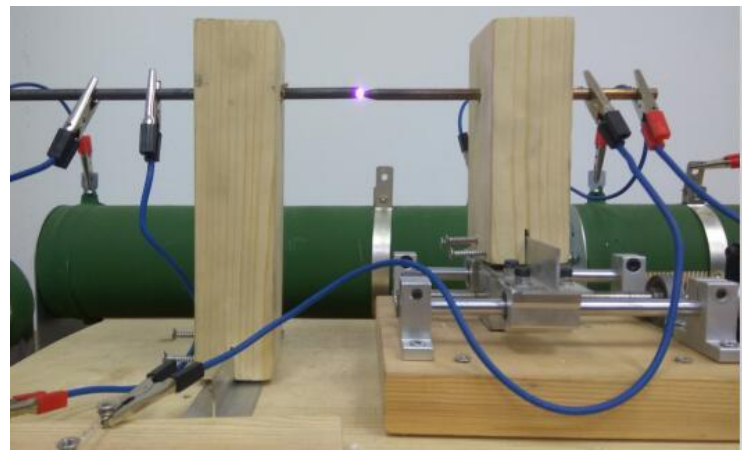

Fig.4 The arc within self-design arc generator 


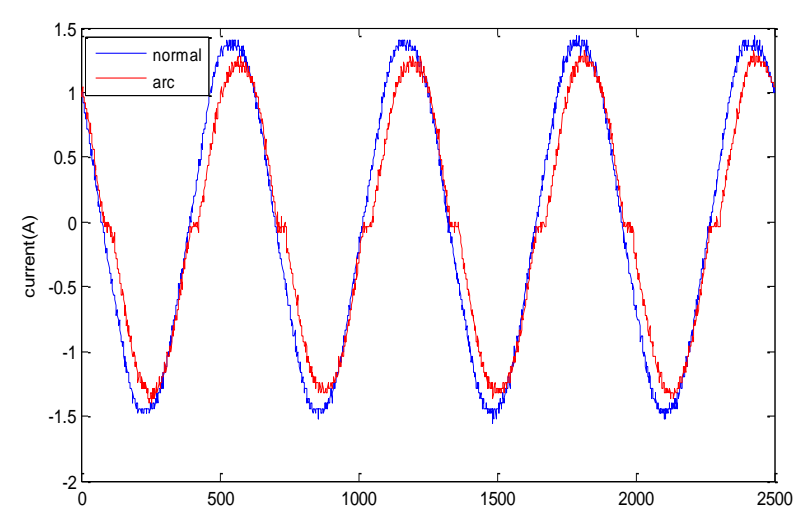

Fig.5 The current waveform at $115 \mathrm{~V} 1 \mathrm{~A}$

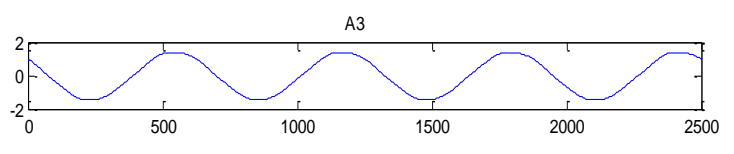

D3
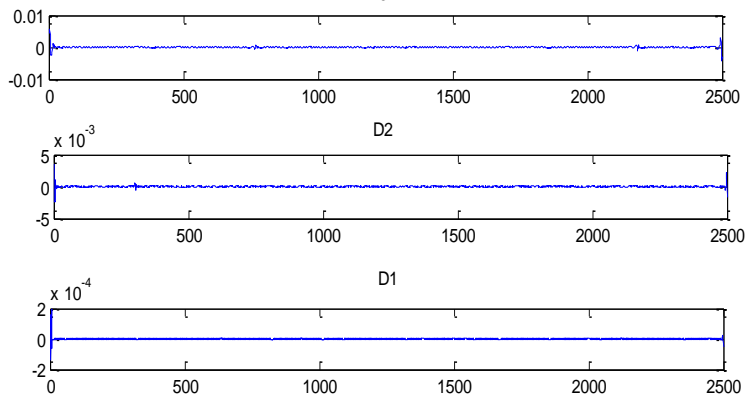

Fig.6 (1) The WT of current in normal circuit
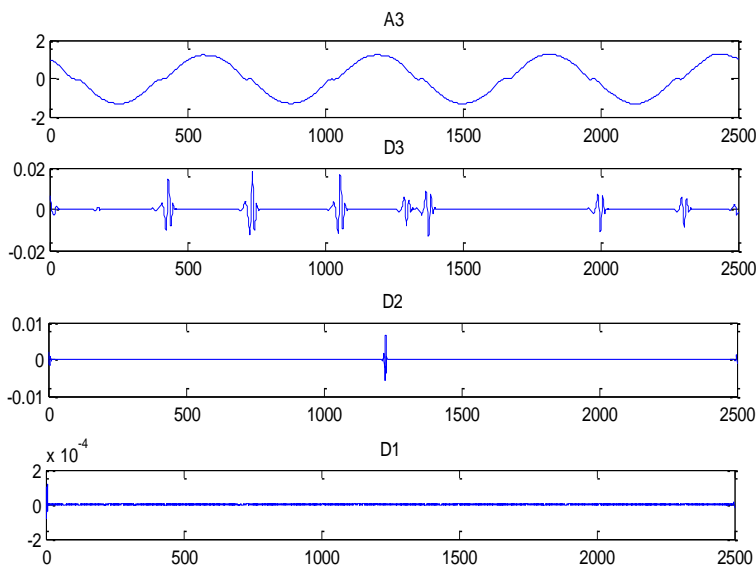

Fig.6 (2) The WT of current in arc fault circuit

In the Fig. 5, it is shown that the most of the arc current waveform is similar to the no-arc current but there are some arc characteristics such as shoulders when the current passes zero, reduced current amplitude compared with no-arc current. As it is mentioned in the previous, A3 component is the approximate component which is similar to the original current signal. D3, D2 and D1 components are the detail components reflecting the high frequency signal. The frequency-band range is as follows:

Table 1 The frequency bands of every reconstructing signals

Reconstructing signal

$\mathrm{D}_{3}$

$\mathrm{D}_{2}$

$\mathrm{D}_{1}$

$\mathrm{A}_{3}$
Frequency bands $(\mathrm{Hz})$

$625 \sim 1250$

1250 2500

2500 5000

$0 \sim 625$

In the Fig. 6(1)-Fig. 6(2), it is obviously that arc fault circuit has higher D3 component value than its in normal circuit and the D3 component have a large mutation during the current passing zero or large current period. However, the values of D2 and D1 components are near zero except for some large current point during the arc. While the D3, D2 and D1 components during no-arc period are near zero. So the D3 component can be a characteristic of fault arc. In the Table 1, the D3 frequency-band range is $625 \sim 1250 \mathrm{~Hz}$, which the arc fault current has high frequency component, while the normal current is $400 \mathrm{~Hz}$. Because of the wavelet energy is solved by the detail components, the regular of wavelet energy is the same with D3 component in Fig. 7. 


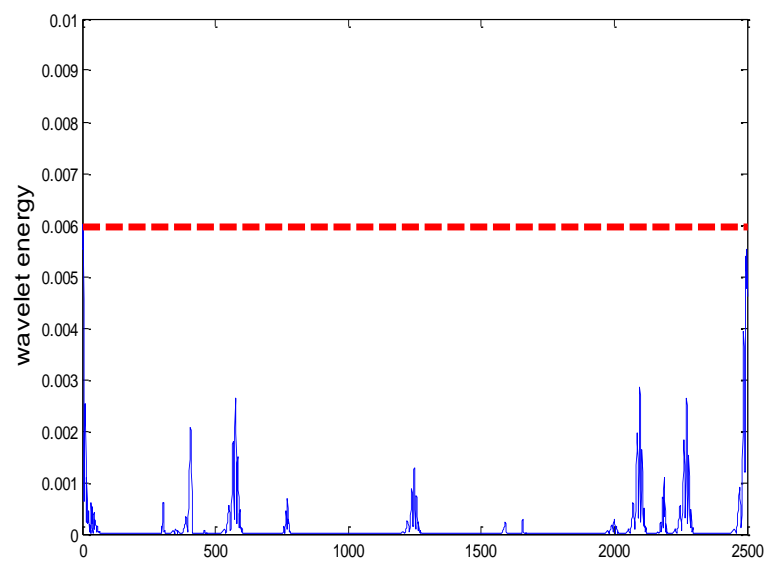

Fig. 7(1) The WE of fault normal current

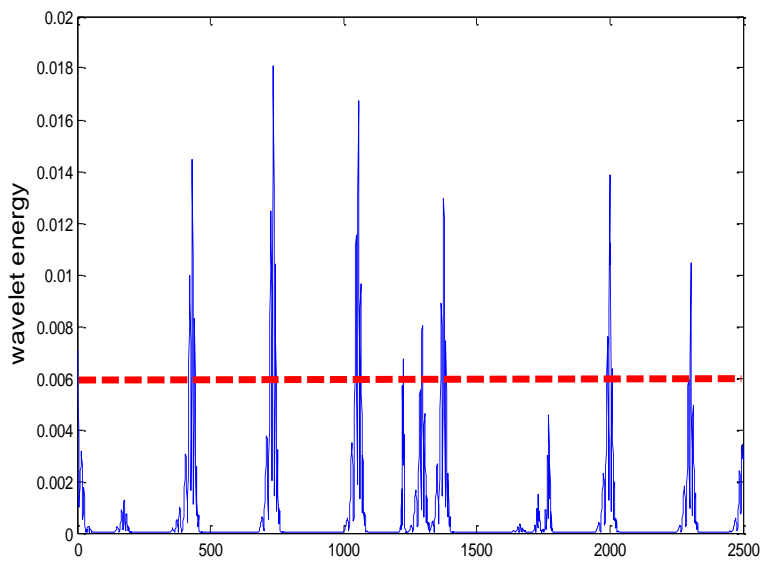

Fig.7 (2) The WE of fault arc current

Compared with different loads current, the wavelet energy changes with the load current changing. The larger the current is, the bigger wavelet energy it has. Though normalization, which the wavelet energy divide the no-arc load current, the wavelet energy of no-arc circuit is lower than 0.006, so the wavelet energy threshold is set as 0.006 . In order to distinguish the arc from normal current accurately, the information entropy method is applied. The calculation results of information entropy and characteristic frequency-band are shown in Table 2.

Table 2 The comparison of the characteristic of series arc

\begin{tabular}{cccc}
\hline$U(\mathrm{~V})$ & $I(\mathrm{~A})$ & $I E$ & $F B(\mathrm{~Hz})$ \\
\hline & 1 & 2.021 & \\
\multirow{2}{*}{115} & 5 & 1.835 & $625 \sim 1250$ \\
& 10 & 2.12 & \\
\hline
\end{tabular}

The results show that the $I E$ of arc current is bigger than that of no-arc current, and the $I E$ has nothing to do with the load current. The $I E$ of arc current is bigger than 1 . While the $I E$ of no-arc current is lower than 0.5 , so the threshold is set as 1 . The large $I E$ value appears in the current passing zero region.

\section{Conclusion}

In this paper, a simulation experiment is carried out to simulate the $\mathrm{AC}$ series arc fault and a united method of the wavelet transform and the information entropy is proposed to distinguish the fault and normal circuit. Comparing the analysis results, it can be seen that the fault arc characteristics as follows:

1) The fault arc frequency bands range is $(625,1250) \mathrm{Hz}$;

2) D3 component and wavelet energy have big value and the wavelet energy is bigger than 0.006 .

3) The current of fault circuit has shoulders when the current pass zero.

4) The current of fault circuit is lower than the no-arc current.

5) The $I E$ of fault circuit is bigger than 1.

If the detected circuit has all or most of these characteristics, the series fault arc maybe take place.

\section{Acknowledgments}

This work is supported by the National Natural Science Foundation of China under Grant Nos.51177005 and 51477004.

\section{References}

[1] Li Shi. Study of low cost arc fault circuit interrupter based on MCU. International Journal of Control and Automation. Vol. 8 (2015) No. 10, p. 25-34. 
[2] Grassetti, Riccardo Grassetti, Roberto Ottoboni, et al. Low cost arc fault detection in aerospace applications. IEEE Instrumentation and Measurement Magazine. Vol. 16 (2013) No. 5, p. 37-42.

[3] Malakondaiah Naidu, Thomas J. Schoepf, Suresh Gopalakrishnan. Arc Fault Detection Scheme for 42V Automotive DC Networks Using Current Shunt International. IEEE TRANSACTIONS ON POWER ELECTRONICS. Vol. 21 (2006) No.3, p. 633-639.

[4] K Yang, R Zhang, J Yang, Y Chen, et al. Research on Low-voltage Series Arc Fault Detection Method Based on Least Squares Support Vector Machine. Open Electrical \& Electronic Engineering Journal, Vol. 9 (2015) No.1, p. 408-421.

[5] George D. Gregory, Kon Wong, Robert F. Dvorak. More about Arc-Fault Circuit Interrupters. IEEE TRANSACTIONS ON INDUSTRY APPLICATIONS. Vol. 40 (2004), No.4, p. 1006-1011.

[6] Huang Yuanhang, Wang Yongxing, Dong Enyuan, et al. Aviation Arc Fault Diagnosis Based on Weight Direct Determined Neural Network. 2nd International Conference on Electric Power Equipment-Switching Technology. Matsue, Japan, October 20-23 2013, p. 1-4.

[7] Xiaoming Liu, Xiangning Liu, Chunguang Hou, et al. Arc Fault Diagnosis and Analysis Based on Wavelet Neural Network. 1st International Conference on Electric Power Equipment- Switching Technology. Xi'an, China, October 23-27 2011, p. 187-190.

[8] Hongkun Zhang, Tao Chen. Arc Fault Signatures Detection on Aircraft Wiring System. The 6th World Congress on Intelligent Control and Automation. Dalian, China, June 21-23 2006, p. $5548-5552$.

[9] UL1699, UL Standard for Safety for Arc-Fault Circuit-Interrupters.

[10] AS5692, ARC Fault Circuit Breaker (AFCB), Aircraft, Trip-Free Single Phase and Three Phase 115 VAC, 400 Hz-Constant Frequency.

[11]He Kuanfang, Wu Jigang, Li Xuejun. Wavelet Analysis for Electronic Signal of Submerged Arc Welding Process. Third International Conference on Measuring Technology and Mechatronics Automation. Shanghai, China, January 6-7 2011, p. 1139-1141.

[12] Silvano Vergura; Mario Carpentieri; Vito Puliafito. A time-frequency analysis of electrical users by means of Fourier and Wavelet transforms [J]. 16th International Conference on Environment and Electrical Engineering (EEEIC). Florence, Italy, June 7 2016, p. 1-6.

[13]Zhan Wang, Stephen McConnell, Robert S. Balog, et al. Arc Fault Signal Detection-Fourier Transformation vs. Wavelet Decomposition Techniques using Synthesized Data. IEEE 40th Photovoltaic Specialist Conference (PVSC). Denver, Colorado, USA. June 8-13 June 2014, p. 3239-3244.

[14]Zheng you $\mathrm{He}$, Ling $\mathrm{Fu}$, Sheng Lin, et al. Fault Detection and Classification in EHV Transmission Line Based on Wavelet Singular Entropy. IEEE TRANSACTIONS ON POWER DELIVERY, Vol.25, (2010) No.4, p. 2156-2163.

[15] Kaiqiang Liang, Zhen Huang, Dexiu Hu, et al. An Individual Emitter Recognition Method Combining Bispectrum with Wavelet Entropy. 2015 IEEE International Conference on Progress in Informatics and Computing (PIC). Nanjing, China. December 18-20 2015, p. 206-210. 